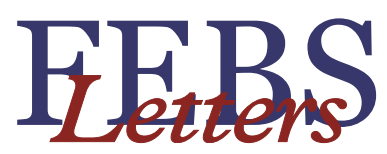

journal homepage: www.FEBSLetters.org

\title{
Molecular basis of the NO trans influence in quaternary T-state human hemoglobin: A computational study
}

\author{
Ariel Alcides Petruk ${ }^{\mathrm{a}}$, Alessandro Vergara ${ }^{\mathrm{b}, \mathrm{c}}$, Dario Estrin ${ }^{\mathrm{a}}$, Antonello Merlino ${ }^{\mathrm{b}, \mathrm{c}, *}$ \\ a Departamento de Química Inorgánica, Analítica y Química Física/INQUIMAE-CONICET, University of Buenos Aires, Buenos Aires, Argentina. \\ ${ }^{\mathrm{b}}$ Department of Chemical Sciences, University of Naples "Federico II", via Cintia, 80126 Napoli, Italy. \\ ' Institute of Biostructures and Bioimages, CNR, via Mezzocannone 16, 80100 Napoli, Italy.
}

\section{A R T I C L E I N F O}

\section{Article history:}

Received 16 April 2013

Revised 31 May 2013

Accepted 3 June 2013

Available online 13 June 2013

Edited by Stuart Ferguson

\section{Keywords:}

Nitric oxide

Nitrosylhemoglobin

Molecular dynamics

\begin{abstract}
A B S T R A C T
NO binding to the T-state of human hemoglobin ( $\mathrm{HbA})$ induces the cleavage of the proximal His bonds to the heme iron in the $\alpha$-chains, whereas it leaves the $\beta$-hemes hexacoordinated. The structure of the nitrosylated T-state of the W37E $\beta$ mutant (W37E) shows that the Fe-His87 $\alpha$ bond remains intact. Exactly how mutation affects NO binding and why tension is apparent only in $\mathrm{HbA} \alpha$-heme remains to be elucidated. By means of density functional theory electronic structure calculations and classical molecular dynamics simulations we provide an explanation for the poorly understood NO binding properties of $\mathrm{HbA}$ and its W37E mutant. The data suggest an interplay between electronic effects, tertiary structure and hydration site modifications in determining the tension in the NO-ligated T-state HbA $\alpha$-chain.
\end{abstract}

() 2013 Federation of European Biochemical Societies. Published by Elsevier B.V. All rights reserved.

\section{Introduction}

Nitric oxide (NO) is a signaling molecule that regulates essential physiological processes [1,2]. Endothelium-derived NO regulates vascular tone and inhibits platelet aggregation as well as lipid peroxidation [3-5]. A large fraction of NO that diffuses into the lumen of the blood vessel rapidly reacts with hemoglobin $(\mathrm{Hb})[6,7]$. $\mathrm{Hb}$ interacts with NO forming the various derivatives [8-11]. Under high oxygen pressure NO both oxidizes oxygenated $\mathrm{Fe}(\mathrm{II}) \mathrm{Hb}$ into the ferric species and is itself oxidized to generate nitrate (1).

$\mathrm{Fe}(\mathrm{II}) \mathrm{O}_{2}+\mathrm{NO}=\mathrm{Fe}(\mathrm{III})+\mathrm{NO}_{3}^{-}$

Nitrosylhemoglobin (Hb-NO) is the product of the reaction in which NO adds to ferrous heme iron of deoxyhemoglobin (2).

$\mathrm{Fe}(\mathrm{II})+\mathrm{NO}=\mathrm{Fe}(\mathrm{II}) \mathrm{NO}$

In human $\mathrm{Hb}(\mathrm{HbA})$ Cys93 $\beta$ serves as an additional NO-binding site $[12,13]$. When Cys93 $\beta$ is nitrosylated, the human Hb S-nitrosyl adduct is known as S-nitrosohemoglobin (SNO-HbA) [13]. The biological role of these nitric oxide derivatives of $\mathrm{Hb}$ is not completely understood. However, several possible functional roles have been proposed. It has been suggested that the main physiological effect of $\mathrm{Hb}$ on NO in vivo is to inactivate it [14]. The reduction of NO

* Corresponding author at: Department of Chemical Sciences, University of Naples "Federico II", via Cintia, 80126 Napoli, Italy.

E-mail address: antonello.merlino@unina.it (A. Merlino). activity by $\mathrm{Hb}$ is supported by evidence of vasoconstriction during infusion of Hb-based blood substitutes $[15,16]$. It has also been proposed that NO can function as an allosteric regulator of hemoglobin oxygen-binding properties and that $\mathrm{Hb}$ can store NO [17]. Alternatively, a possible role involving nitrite reductase activity by deoxy-Hb has been hypothesized [18]. Since both SNO-HbA and HbA-NO have been detected in vivo $[13,19]$, it has been suggested that $\mathrm{NO}$ is transported by $\mathrm{Hb}$ as the S-nitrosyl adduct, formed by intramolecular transfer from nitrosyl-HbA $[12,13,20]$.

The stereochemistry of the interaction of NO with the heme is complex [8-11]. It has been shown that the quaternary structure of nitrosyl $\mathrm{Hb}$ can be switched from the R-state quaternary ensemble to the T-state by adding the allosteric effector inositol hexaphosphate [8-10]. X-ray crystallographic [21] as well as spectroscopic studies [22] have demonstrated that NO binding to the heme groups of crystalline wild-type deoxyhemoglobin (Tstate) ruptures the Fe-His bonds in the $\alpha$-chains (Fe-His87 $\alpha$ ). In contrast, the corresponding $\beta$-subunit bond (Fe-His92 $\beta$ ) is not broken [21]. The current explanation of this phenomenon is related to the Perutz hypothesis [23,24], which has been supported by molecular orbital calculations performed by Mingos [25,26], that the formation of the T-state from two $\alpha \beta$-dimers creates constraints at the dimer-dimer interface that resist the ligand-induced movement of the $\alpha$-heme Fe atom into the plane of porphyrin ring [21]. This resistance results in ligand-induced tension on $\mathrm{Fe}-$ His $87 \alpha$, but not on Fe-His92 $\beta$. Most of the quaternary constraints are localized to a small cluster of residues that are centred around 


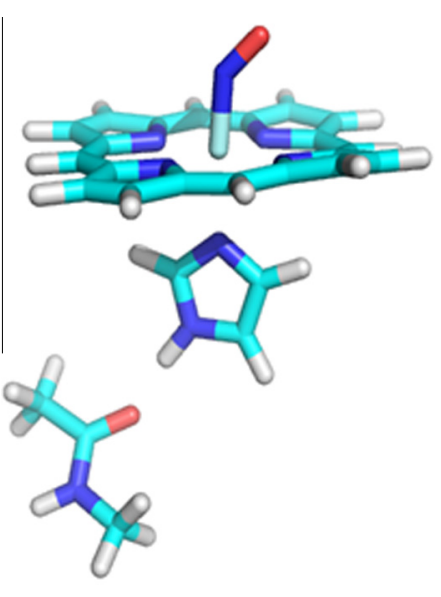

A

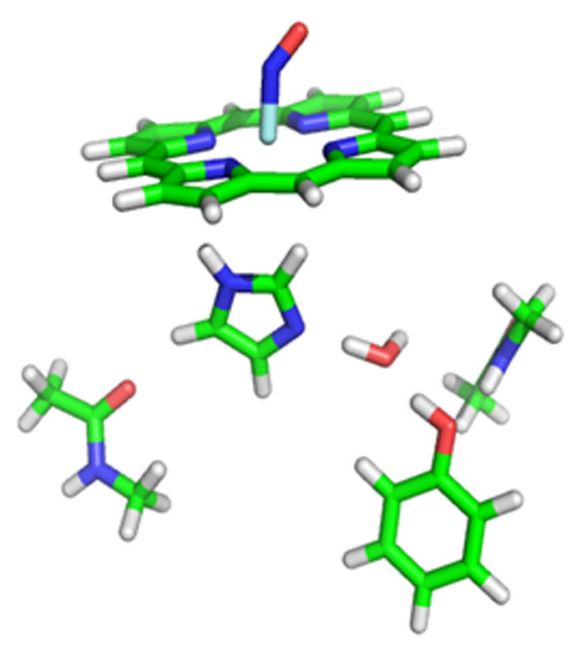

B

Fig. 1. Model systems for HbA heme pocket.

residue $\operatorname{Trp} 37 \beta$. The structure of the nitrosylated T-state of the W37E $\beta$ mutant shows that Fe-His87 $\alpha$ remains intact, thus confirming the Perutz hypothesis [21].

However, it remains to be explained why NO so dramatically affects only $\alpha$-hemes and exactly how the W37E $\beta$ mutation is linked to the loss of tension on the $\alpha$-heme. Knowledge of the structural and dynamic features determining the tension at the $\alpha$-heme is crucial for deciphering the structural determinants of the $\alpha \beta$-dimer communication at the heart of $\mathrm{Hb}$ cooperativity.

During the last decade, we performed several structural and computational studies for different members of the globin superfamily [27-32], with the aim of studying how tertiary structure and dynamics control ligand affinity. To advance in our understanding of the relationship between structural modifications and ligand binding in globins we have analyzed the NO trans influence ${ }^{1}$ in the isolated heme pocket HbA chain model systems by density functional theory (DFT) and have performed classical molecular dynamics (MD) simulations of the nitrosylated forms of $\mathrm{HbA}$ and W37E. Our goal is to provide an explanation of the different behaviors of the $\alpha$ - and the $\beta$-chains of HbA, and of HbA vs W37E. Our results reveal an interplay between the electronic effects, tertiary structure variations and modifications of hydration sites.

\section{Methods}

\subsection{Models and computational methods}

\subsubsection{DFT calculations}

All QM calculations were performed at the density functional theory (DFT) level with the SIESTA code [33]. For all atoms, basis sets of double- $\zeta$ plus polarization quality were employed; all calculations were performed by using the generalized gradient approximation functional proposed by Perdew, Burke, and Ernzerhof (PBE) [34]. For all systems studied, the spin-unrestricted approximation was used. The QM subsystem included the heme group (without the peripheral groups), the NO, the His imidazole group $(87 \alpha$ and $92 \beta)$, and backbone atoms of a Leu residue $(83 \alpha$ and $88 \beta$ ) (Fig. 1A and B). This methodology was proved to be appropri-

\footnotetext{
${ }^{1}$ Trans influence is a change in the bond distance between the metal and a ligand that is trans to that which exerts the effect through the same d-orbital interaction. Trans effect is the effect shown by a ligand whereby it directs the substitution of another ligand placed trans to it. Here we refer to trans influence as the lengthening of the bond trans to the NO.
}

ate for heme proteins, as shown by several works from our group [35-38].

\subsubsection{MD simulations}

To investigate how structure and dynamics of nitrosylated form of HbA and W37E may affect the Fe-His distance, MD simulations were carried out. The initial structures for the MD simulations were built starting from the X-ray structure of nitrosylated $\mathrm{HbA}$ and W37E (PDB codes 1RPS and 1RQA, respectively) [21]. Particular attention was paid to the building of missing coordinates in the mutant structure. In particular, the PDB model of W37E lacks atoms of the side chain of Tyr $140 \alpha$. Superimposition of backbone atoms of Tyr $140 \alpha$ of HbA with the same atoms of W37E reveals that the wild-type conformation of the side chain of Tyr $140 \alpha$ produces serious crashes with backbone or side chain atoms of residue Ser138 $\alpha$, Thr137 $\alpha$ and/or of carbonyl of Asp94 $\alpha$ in the mutant. Since the superposition of the sole backbone atoms of Tyr $140 \alpha$ might not be suitable to properly orient the $\alpha$-chain, we have also aligned the entire alpha chains of the two proteins. After the superimposition, the aromatic ring of Tyr $140 \alpha$ (as in $\mathrm{HbA}$ ) collides with the side chain atoms of Ala88 in W37E (as noted by short contacts close to 2.0-2.2 $\AA$ ). Altogether these findings suggest that Tyr $140 \alpha$ adopts a distinct conformation in the mutant. For this reason we decided to position the side chain of Tyr140 $\alpha$ exposed to the solvent in the mutant (Fig. S1). This hypothesis is in line with the $\mathrm{X}$-ray data showing absence of electron density corresponding to Tyr140 $\alpha$ side chain, thus revealing a large conformation variability for this residue.

Furthermore, to complete the picture of the mutant-induced conformational variation that may affect the iron coordination, two additional simulations, called HbA-to-W37E (HbA2W37E) and W37E-to-HbA (W37E2HbA), have been performed. The first simulation, called HbA2W37E, corresponds to a MD simulation starting from the structure of $\mathrm{HbA}$, in which $\operatorname{Trp} 37 \beta$ was replaced with Glu. The second simulation, called W37E2HbA, corresponds to a MD study starting from the structure of the mutant, in which Glu37 $\beta$ was replaced with Trp. Finally, an additional simulation has been performed, called HbA2W37E_Yout, which corresponds to a MD study starting from the structure of $\mathrm{HbA}$, in which Trp37 $\beta$ have been replaced by Glu and Tyr140 $\alpha$ occupies a position exposed to the solvent. All simulations were carried out by using the AMBER 11 suite of programs and AMBER parm99 force field [39]. The heme parameters were the same used for our previous works [35-38], but all the Fe-NE2(His) bond force constants were set to $0 \mathrm{kcal} /$ 
$\mathrm{mol} / \AA^{2}$. The system energy was first minimized by steepest descent algorithm followed by the conjugate gradient algorithm giving a total of 12000 steps.

Harmonic restraints applied to the $\alpha$-carbons were slowly relaxed from 25 to $1 \mathrm{kcal} / \mathrm{mol} / \AA^{2}$ by the end of the energy minimization. To save computational time, a 10 - $\AA$ cutoff for non-bonded interactions was used. The equilibration period in the molecular dynamics (MD) simulations consisted of eight stages. In the first one, the system was gradually heated to $300 \mathrm{~K}$ for $50 \mathrm{ps}$ at $50 \mathrm{~K}$ intervals followed by $50 \mathrm{ps}$ at $300 \mathrm{~K}$. The remaining restraints were gradually reduced to zero in this stage. The second stage consisted of $50 \mathrm{ps}$ of unrestrained equilibration. Solute temperature was weakly coupled to a Berendsen temperature bath [40] at $300 \mathrm{~K}$ with a time constant of 2 ps. Bond lengths including hydrogens were constrained by using the SHAKE algorithm [41]. The time step was 2 fs. The center-of-mass motion was removed at regular 1-ps intervals. A simulation of $10 \mathrm{~ns}$ for each protein system has been performed.

\section{Results and discussion}

\subsection{DFT calculations}

The comparison between the NO-bound structures of $\mathrm{HbA}$ and W37E shows that a significant difference in the heme pocket is the presence of a water molecule that forms hydrogen bonds with Tyr140 $\alpha$ and Val93 $\alpha$ (Fig. 2). This water molecule, hereafter denoted as the bridging water, has not been observed in the $\beta$-heme pocket and it is not conserved in the structure of the nitrosylated Tform of the W37E mutant. In the latter, the binding of NO does not induce the rupture of the His-Fe bond, but provokes an increase in the flexibility of the surrounding region, with the Tyr140 $\alpha$ that becomes invisible in the electron density maps.

We have first considered isolated model systems consisting of the heme (excluding the propionate and vinyl groups), proximal His imidazole ( $87 \alpha$ and $92 \beta)$ and backbone atoms of a Leu residue $(83 \alpha$ and $88 \beta)$ that are in contact with proximal His side chain atoms (Fig. 1A) using atomic coordinates from $\beta$-chains of $\mathrm{HbA}$, $\alpha$ - and $\beta$-chains of W37E. Selected structural parameters obtained from the optimized structures are presented in Table 1.

We observed a subtle variation of the Fe-His distance, which increases about $0.05-0.14 \AA$, relative to that of the isolated model of deoxy $\mathrm{HbA}$, in NO-bound heme models of both $\alpha$ - and $\beta$-chains (Ta- ble 1). This finding is in line with the NO trans influence, i.e. with a weakening of Fe-His bond. However, the reported values do not justify the observed rupture of the Fe-His bond in $\mathrm{HbA} \alpha$-chains, since the bond rupture corresponds to an increase of the distance $>1 \AA$ A. This indicates that Fe-His bond breakage depends not only on the Fe-His bond strength but also on other factors.

Because surrounding residues often play important roles in ligand binding, we decided to perform a geometric characterization of an extended model system composed of NO-bound $\alpha$-heme with iron not coordinated to imidazole of His $87 \alpha$, backbone atoms of Leu83 $\alpha$ and Val93 $\alpha$, the bridging water and the Tyr $140 \alpha$ side chain (Fig. 1B). The results of this analysis point out a critical role of the Tyr $140 \alpha$ side chain and of bridging water in stabilizing the pentacoordinated form: the value obtained for the Fe-His distance, in fact, is on average equal to $3.95 \AA$ (Table 2).

\subsection{Molecular dynamics simulations}

Based on these data, we surmised that Tyr140 $\alpha$ is responsible for holding the bridging water in the position needed to interact with proximal His side chain (Fig. 2). The perturbation of the Tyr140 $\alpha$ conformation induced by the W37E mutation should explain the different behaviour of the mutant compared to HbA. Moreover, larger flexibility of Tyr145 $\beta$ (also due to the absence of a residue corresponding to $\mathrm{W} 37 \beta$ in the $\alpha$-chain) with respect to that observed for Tyr140 $\alpha$ should explain the different behavior of the $\alpha$ - and $\beta$-HbA chains.

To validate these hypotheses and better understand the role of Tyr140 $\alpha$ and of the bridging water in the breakage of His-Fe bond, we have carried out $10 \mathrm{~ns}$ MD simulations of the following systems: HbA, W37E, HbA2W37E, W37E2HbA and HbA2W37E_Yout, not including the Fe-His covalent bond in the force field (see Section 2). We first analyzed the stability of the simulations by computing the root mean square deviations (RMSd) from the starting structure as function of time (Fig. S2). The results of this analysis show that the structures of HbA, HbA2W37E and W37E are quite stable during the simulations (RMSd $<1.5 \AA$ ), whereas those of HbA2W37E_Yout and W37E2HbA present large RMSd values (about $3 \AA$ ). To assess if the large RMSd observed in the cases of HbA2W37E_Yout and W37E2HbA are due to a quaternary structure transition, we have calculated the angle needed to superimpose the $\alpha 2 \beta 2$ dimer of each simulated system to the same region of deoxy $\mathrm{HbA}$ (PDB code $2 \mathrm{HHB}$ ) after the superimposition

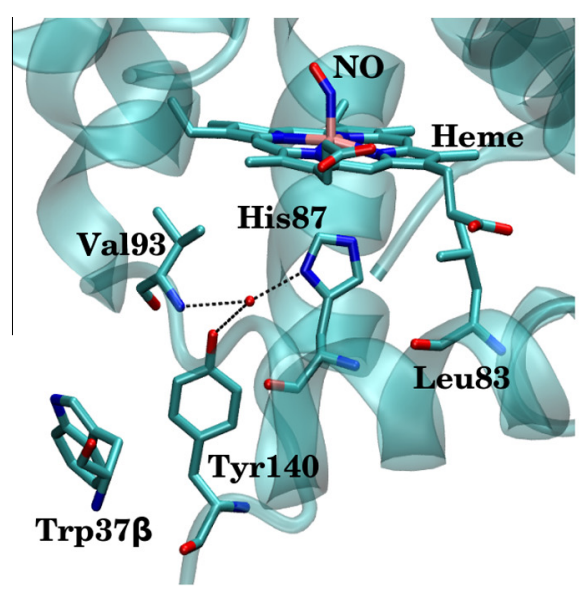

A

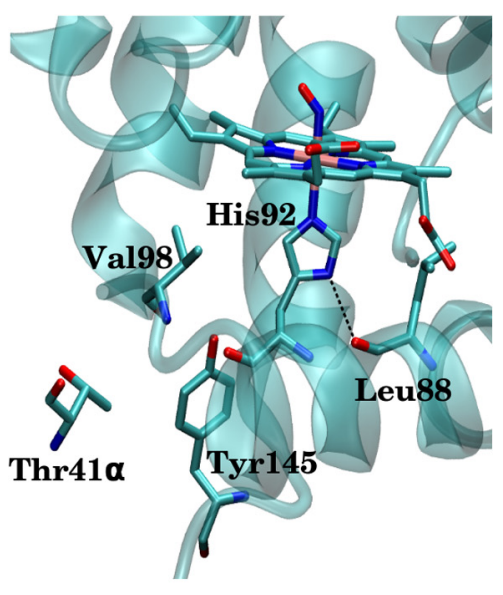

B

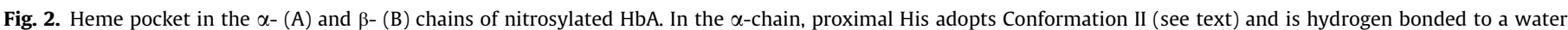

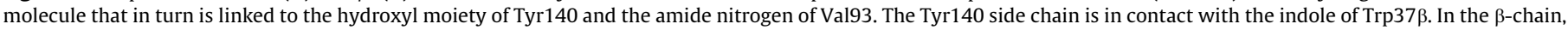
the His adopts Conformation I, in which it forms a hydrogen bond with the backbone carbonyl of Leu88. 
Table 1

Structural optimized parameters for the NO hexacoordinated complexes in vacuum.

\begin{tabular}{|c|c|c|c|c|c|c|}
\hline Starting coordinates & HbA_ß1 & HbA_ß2 & W37E_ $\alpha 1$ & W37E_ $\alpha 2$ & W37E_ $\beta 1$ & W37E_ $\beta 2$ \\
\hline $\mathrm{Fe}-\mathrm{NO}$ & 1.754 & 1.760 & 1.754 & 1.756 & 1.756 & 1.748 \\
\hline $\mathrm{d}(\mathrm{Fe}-\mathrm{NeHis})$ in pentacoordinated isolated model system & 2.160 & & & & & \\
\hline $\mathrm{d}(\mathrm{Fe}-\mathrm{NeHis})$ & 2.240 & 2.230 & 2.252 & 2.216 & 2.272 & 2.308 \\
\hline$<$ NeHis-Fe-N1 $\left({ }^{\circ}\right)$ & 87.883 & 87.722 & 87.387 & 87.892 & 87.701 & 86.025 \\
\hline$<\mathrm{NeHis}-\mathrm{Fe}-\mathrm{N} 2\left(^{\circ}\right)$ & 86.493 & 88.874 & 85.307 & 86.774 & 87.262 & 86.330 \\
\hline$<$ NeHis-Fe-N3 $\left(^{\circ}\right)$ & 86.325 & 87.318 & 88.378 & 87.432 & 86.601 & 86.825 \\
\hline$<$ NeHis-Fe-N4 $\left(^{\circ}\right)$ & 86.954 & 86.179 & 89.785 & 88.890 & 86.662 & 85.672 \\
\hline d N ...O (Distances are in $\AA$ ) & 2.750 & 2.741 & 2.818 & 2.778 & 2.769 & 2.782 \\
\hline
\end{tabular}

Table 2

Structural optimized parameters for the NO pentacoordinated complexes in vacuum.

\begin{tabular}{|c|c|c|}
\hline Sarting coordinates & HbA_ $\alpha 1$ & HbA_ $\alpha 2$ \\
\hline Fe-NO & 1.734 & 1.730 \\
\hline $\mathrm{d}(\mathrm{Fe}-\mathrm{NeHis})$ & 3.939 & 3.967 \\
\hline $\mathrm{d}(\mathrm{Fe}-\mathrm{NdHis})$ & 5.066 & 5.074 \\
\hline $\mathrm{d}(\mathrm{Nim}-\mathrm{OH} 2)$ & 2.936 & 2.638 \\
\hline $\mathrm{d}(\mathrm{O}$ Wat-OTyr) & 2.698 & 2.578 \\
\hline $\mathrm{d}(\mathrm{O}$ Wat-N) (Distances are in $\AA$ ) & 3.095 & 3.523 \\
\hline
\end{tabular}

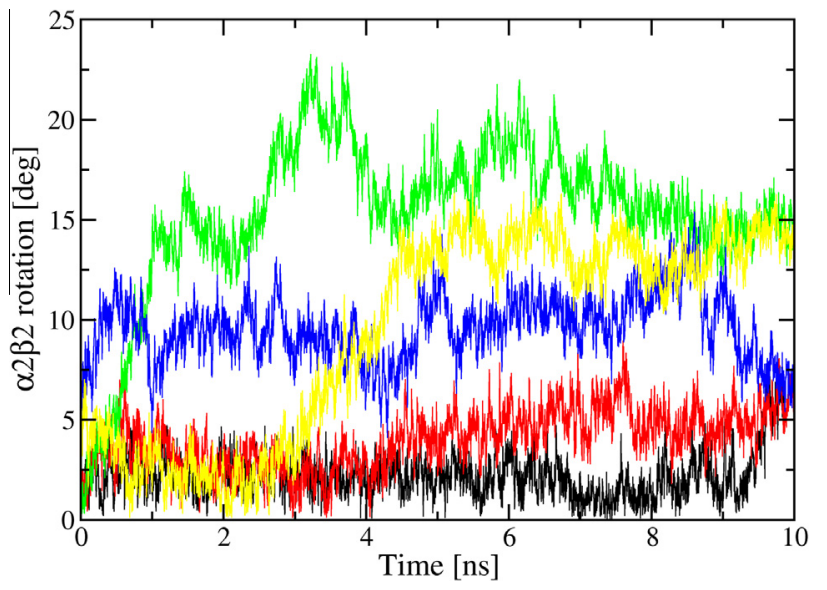

Fig. 3. Rotation of the $\alpha 2 \beta 2$ dimer with respect to the $\alpha 1 \beta 1$ dimer during the simulations. HbA (black), HbA2W37E (red), HbA2W37E_Yout (green), W37E (blue) and W37E2HbA (yellow).

of $\alpha 1 \beta 1$ (Fig. 3). The results of this analysis suggest that $\mathrm{HbA}$ and HbA2W37E retain the T-state quaternary structure, whereas HbA2W37E_Yout, W37E and W37E2HbA shift towards a R-like state. These data are consistent with a number of experimental data showing that the constraints at the $\alpha 1 \beta 2$ interface play a role in triggering the T-to-R transition $[42,43]$.

Previous (and unpublished $100 \mathrm{~ns}$ ) MD studies starting from $\mathrm{T}$ state do not show any quaternary structure conformational variation in the time-scale analyzed here $[44,45]$. Thus, our data indicate that the "Y out" conformation (found in the starting models of HbA2W37E_Yout, W37E and W37E2HbA) accelerates the process. In our opinion, these findings suggest that the mobility of Y140 $\alpha$ could have an important role in the quaternary T-to-R-like transition. Notably, the replacement of W37 $\beta$ with smaller residues Gly (Hb Howick) [43] or Ser (Hb Hirose) [42] increases the oxygen affinity.

\subsection{Looking for the structural determinants of Fe pentacoordination}

We now turn our attention to the Fe-His coordination. First we evaluated the Fe-His distance in the five simulations. The histo- grams reporting the normalized population of Fe-His distances in the $\alpha$ - and $\beta$-chains of all the studied systems are shown in Fig. 4.

In $\mathrm{HbA}$, the distribution of Fe-His distances in the $\beta$-chains and in the $\alpha 2$-chain remain very close to their respective starting values, whereas the histogram of Fe-His distances is broader for the $\alpha 1$-chain, spanning the short distances sampled by the $\beta$-chains as well as the longer distances observed for the $\alpha 2$-chain (Fig. 4A). Interestingly, the W37E mutation results in a narrowing of this Fe-His distance distribution in the $\alpha 1$-chain around shorter values for HbA2W37E (Fig. 4B). For the alternative "Y out" conformation of the mutant (HbA2W37E_Yout) we observed a similar shortening of the Fe-His distance not only in the $\alpha 1$-chain, but also in the $\alpha 2$-chain (Fig. 4C). These data indicate that the Fe-His distance in the $\mathrm{HbA} \alpha 2$-chain is larger than that expected for a standard coordination bond, whereas the values observed for the $\mathrm{HbA} \beta$-chains, and to some extent for the $\mathrm{HbA} \alpha 1$-chain, are in line with the presence/formation of the bond. In W37E and W37E2HbA (Fig. 4D and E, respectively), the Fe-His distances are maintained near the already short starting values $(2.5 \AA)$. In summary, we observed that in $\alpha 1$-chain of HbA, the Fe-His distance has a higher tendency to fluctuate towards shorter lengths than in the $\alpha 2$-chain, and that this tendency is enhanced by the W37E mutation and is further adopted by the $\alpha 2$-chain when the alternative outward Tyr conformation is considered.

To analyze the mechanism of Fe-His bond shortening, we followed in detail the behaviour of heme pocket residues. Interestingly, in the $\alpha 1$-chain of both $\mathrm{HbA}$ and HbA2W37E we observed a strict correlation between the movement of Tyr $140 \alpha$ side chain, the positioning of the bridging water molecules, the flipping of proximal His residue and the decrease of the Fe-His distance. These findings are shown by Figs. S3-S5, where we report the time evolution of the distances between the bridging water molecule and either the hydroxyl oxygen of Tyr140 $\alpha$ (Fig. S3) or the imidazole nitrogen (ND1) of His87 (Fig. S4), along with the time evolution of the C-CA-CB-CG dihedral angle of Tyr140 $\alpha$ (Fig. S5). These data clearly show that when Tyr140 $\alpha$ changes its conformation, water can no longer be tightly hydrogen bonded to His $87 \alpha$. The absence of the bridging water favors the flipping of His87 $\alpha$ side chain, allowing it to form a hydrogen bond with the carbonyl of Leu83 $\alpha$ and to coordinate to the heme iron. Similar results were obtained in HbA2W37E_Yout. The evolution of the $\chi_{1}$ dihedral angle (CA-CB-CG-ND1) of His87 as function of time for the five simulations is reported in Fig. S6. As expected, the His side chain can adopt two different conformations: one with $\chi_{1}$ close to $90^{\circ}$ (Conformation I), where the imidazole can form the hydrogen bond with the carbonyl of Leu ( $83 \alpha$ or $88 \beta$, as in Fig. 2B), and another with $\chi_{1}$ close to $-90^{\circ}$ (Conformation II, Fig. 2A). Simulations starting with Conformation I (i.e. W37E, W37E2HbA) maintain this conformation, and, except for the $\alpha 2$-chain of $\mathrm{HbA}$, the other simulations (HbA, HbA2W37E and HbA2W37E_Yout) adopt this conformation after a few nanoseconds, suggesting that Conformation I is more favorable. Conformation I also favours the shortening of the Fe-His distance. The outward swing of Tyr140 $\alpha$ drives the proximal His to this conformation. 

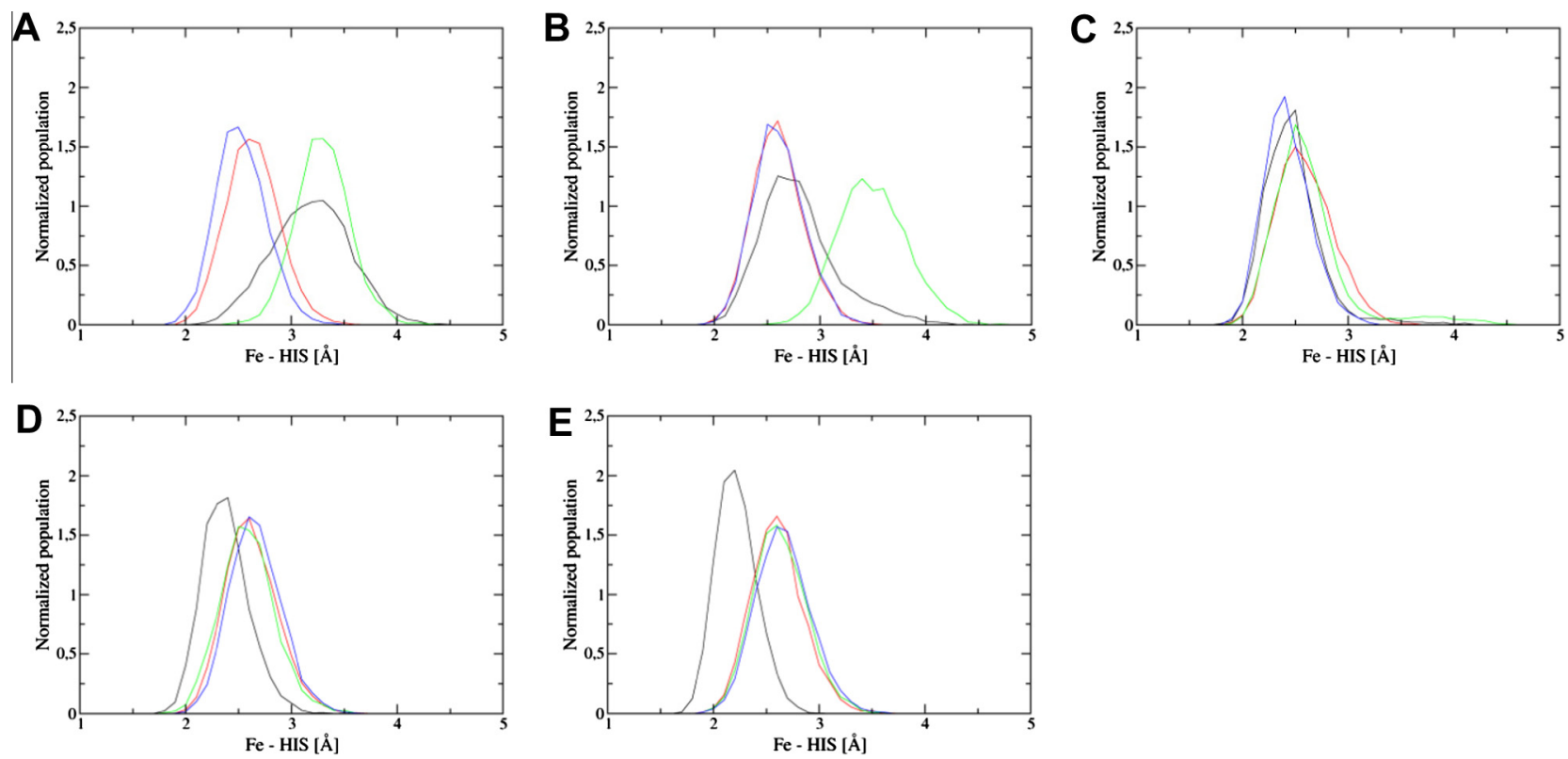

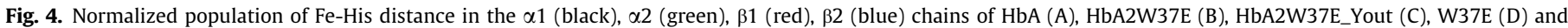
W37E2HbA (E).

Altogether these findings indicate that the conformational changes of Tyr $140 \alpha$ correlate well with lower Fe-His distances. Specifically, the inward conformation of the Tyr140 $\alpha$ side chain, which is favoured by the presence of W37 $\beta$, Thr137 $\alpha$ and Phe98 $\alpha$ in $\mathrm{HbA}$, and the presence of the bridging water molecule in the heme pocket together stabilize Conformation II of the proximal His that, in turn, favours the Fe-His bond breakage (Fig. S7A). In the $\beta$-chain of HbA, Tyr145 is not biased inward, due to the lack of a residue corresponding to $\operatorname{Trp} 37 \beta$ in the $\alpha$-chain at the $\alpha \beta$ interface and due to the replacement of the residue corresponding to the Thr137 $\alpha$ with an Ala (Ala142 $\beta$ ) (Fig. S7B). These data also explain the behaviour of NO binding to the T-state of other Hbs, like those from the Antarctic fish Trematomus bernacchii (HbTb), which shows hexacoordination in both $\alpha$-and $\beta$-chains [46]. In fact, $\mathrm{HbTb}$ exhibits a more variable solvent-exposed conformation of Tyr141 $\alpha$ (and of Tyr145 $\beta$ ), due to its Thr137 $\alpha$-to-Ala replacement (Gly in the $\beta$-chain) and to a slightly different conformation of Phe99 $\alpha$ and positioning of W37 $\beta$ (Fig. S7C and D).

\section{Conclusions}

Using computational techniques we studied the molecular basis for the differential NO binding behaviour of $\alpha$ - and $\beta$-chains in HbA. Our results suggest that a subtle balance of electronic effects, tertiary structure changes, and protein conformational flexibility must be invoked to explain the experimentally observed NO binding characteristics of $\mathrm{HbA}$ and W37E. We found that in the $\alpha$ chains of $\mathrm{HbA}$, the His-Fe bond breakage induced by NO binding is facilitated by the NO trans influence and stabilized by the presence of a water molecule interacting with the proximal His and held in its position by fixed side chain conformation of Tyr140 $\alpha$. Higher flexibility of Tyr140 $\alpha$ in W37E and of the corresponding residue (Tyr145) in the $\beta$-chain of $\mathrm{HbA}$, on the other hand, favors the NO hexacoordination.

\section{Acknowledgements}

The authors thank Marcelo A. Martì for helpful discussion and Mernoosh Arrar for critical reading of the manuscript. AAP is grate- ful to CONICET for a Post-doctoral Fellowship. DE is member of CONICET. Computer power was generously provided by CeCAR at FCEN-UBA and SimOne at INSIBIO-CONICET. AM and AV are grateful to PNRA for financial support.

\section{Appendix A. Supplementary data}

Supplementary data associated with this article can be found, in the online version, at http://dx.doi.org/10.1016/j.febslet.2013. 06.006.

\section{References}

[1] Stamler, J.S., Singel, D.J. and Loscalzo, J. (1992) Biochemistry of nitric oxide and its redox-activated forms. Science 258, 1898-1902.

[2] Gladwin, M.T. and Kim-Shapiro, D.B. (2012) Vascular biology: nitric oxide caught in traffic. Nature 491, 344-345.

[3] Tomasian, D., Keaney, J.F. and Vita, J.A. (2000) Antioxidants and the bioactivity of endothelium-derived nitric oxide. Cardiovasc. Res. 47, 426-435.

[4] Radomski, M.W., Palmer, R.M. and Moncada, S. (1990) Characterization of the L-arginine/nitric oxide pathway in human platelets. Br. J. Pharmacol. 101, 325328.

[5] Pawloski, J.R., Swaminathan, R.V. and Stamler, J.S. (1998) Cell-free and erythrocytic S-nitrosohemoglobin inhibits human platelet aggregation. Circulation 97, 263-267.

[6] Gladwin, M.T., Crawford, J.H. and Patel, R.P. (2004) The biochemistry of nitric oxide, nitrite, and hemoglobin: role in blood flow regulation. Free Radic. Biol. Med. 36, 707-717.

[7] Gladwin, M.T., Lancaster Jr., J.R., Freeman, B.A. and Schechter, A.N. (2003) Nitric oxide's reactions with hemoglobin: a view through the SNO-storm. Nat. Med. 9, 496-500.

[8] Maxwell, J.C and Caughey, W.S. (1976) An infrared study of nitric oxide bonding to heme B and hemoglobin A. Evidence for inositol hexaphosphate induced cleavage of proximal histidine to iron bonds. Biochemistry $15,388-$ 396.

[9] Szabo, A. and Perutz, M.F. (1976) Equilibrium between six- and fivecoordinated hemes in nitrosylhemoglobin: interpretation of electron spin resonance spectra. Biochemistry 15, 4427-4428.

[10] Hille, R., Olson, J.S. and Palmer, G. (1979) Spectral transitions of nitrosyl hemes during ligand binding to hemoglobin. J. Biol. Chem. 254, 12110-12120.

[11] Arcovito, A. and Della Longa, S. (2011) Ligand binding intermediates of nitrosylated human hemoglobin induced at low temperature by X-ray irradiation. Inorg. Chem. 50, 9423-9429.

[12] Bonaventura, J. and Lance, V. (2001) Nitric Oxide, invertebrates and hemoglobin. Amer. Zool. 41, 346-359.

[13] Jia, L., Bonaventura, C., Bonaventura, J. and Stamler, J.S. (1996) Snitrosohaemoglobin: a dynamic activity of blood involved in vascular control. Nature 380, 221-226. 
[14] Eich, R.F. et al. (1996) Mechanism of NO-induced oxidation of myoglobin and hemoglobin. Biochemistry 35, 6976-6983.

[15] Cabrales, P., Han, G., Nacharaju, P., Friedman, A.J. and Friedman, J.M. (2011) Reversal of hemoglobin-induced vasoconstriction with sustained release of nitric oxide. Am. J. Physiol. Heart Circ. Physiol. 300, H49-H56.

[16] Wang, D. et al. (2013). In vivo reduction of cell-free methemoglobin to oxyhemoglobin results in vasoconstriction in canines. Transfusion doi: http:// dx.doi.org/10.1111/trf.12162. [Epub ahead of print].

[17] Stepuro, T.L. and Zinchuk, V.V. (2006) Nitric oxide effect on the hemoglobinoxygen affinity. J. Physiol. Pharmacol. 57, 29-38.

[18] Jensen, F.B. (2009) The dual roles of red blood cells in tissue oxygen delivery: oxygen carriers and regulators of local blood flow. J. Exp. Biol. 212, 33873393.

[19] Funai, E.F., Davidson, A., Seligman, S.P. and Finlay, T.H. (1997) Snitrosohemoglobin in the fetal circulation may represent a cycle for blood pressure regulation. Biochem. Biophys. Res. Commun. 239, 875-877.

[20] Gow, A.J. and Stamler, J.S. (1998) Reactions between nitric oxide and haemoglobin under physiological conditions. Nature 391, 169-173.

[21] Chan, N.L., Kavanaugh, J.S., Rogers, P.H. and Arnone, A. (2004) Crystallographic analysis of the interaction of nitric oxide with quaternary- $T$ human hemoglobin. Biochemistry 43, 118-132.

[22] Szabo, A. and Barron, L.D. (1975) Letter: resonance Raman studies of nitric oxide hemoglobin. J. Am. Chem. Soc. 97, 660-662.

[23] Perutz, M.F., Kilmartin, J.V., Nagai, K., Szabo, A. and Simon, S.R. (1976) Influence of globin structures on the state of the heme. Ferrous low spin derivatives. Biochemistry 15, 378-387.

[24] Perutz, M.F. (1979) Regulation of oxygen affinity of hemoglobin. Annu. Rev. Biochem. 48, 327-386.

[25] Mingos, D.M.P. (1973) A general bonding model for linear and bent transition metal-nitrosyl complexes. Inorg. Chem. 12, 1209-1211.

[26] Hoffmann, R.M., Chen, M.L., Elian, M., Rossi, A.R. and Mingos, D.M.P. (1974) Pentacoordinate nitrosyls. Inorg. Chem. 13, 2666-2675.

[27] Boechi, L., Marti, M.A., Vergara, A., Sica, F., Mazzarella, L., Estrin, D.A. and Merlino, A. (2011) Protonation of histidine 55 affects the oxygen access to heme in the alpha chain of the hemoglobin from the Antarctic fish Trematomus bernacchii. IUBMB Life 63, 175-182.

[28] Balsamo, A., Sannino, F., Merlino, A., Parrilli, E., Tutino, M.L., Mazzarella, L. and Vergara, A. (2012) Role of the tertiary and quaternary structure in the formation of bis-histidyl adducts in cold-adapted hemoglobins. Biochimie 94 , 953-960.

[29] Merlino, A., Vergara, A., Sica, F., Aschi, M., Amadei, A., Di Nola, A. and Mazzarella, L. (2010) Free-energy profile for CO binding to separated chains of human and Trematomus newnesi hemoglobin: insights from molecular dynamics simulations and perturbed matrix method. J. Phys. Chem. B 114 7002-7008.

[30] Arroyo-Manez, P. et al. (2011) Protein dynamics and ligand migration interplay as studied by computer simulation. Biochim. Biophys. Acta 1814 1054-1064.

[31] Bikiel, D.E. et al. (2006) Modeling heme proteins using atomistic simulations. Phys. Chem. Chem. Phys. 8, 5611-5628.
[32] Capece, L., Boechi, L., Perissinotti, L.L., Arroyo-Manez, P., Bikiel, D.E. Smulevich, G., Marti, M.A. and Estrin, D.A. (2013) Small ligand-globin interactions: reviewing lessons derived from computer simulation. Biochim. Biophys. Acta. doi: http://dx.doi.org/10.1016/j.bbapap.2013.02.038. [Epub ahead of print].

[33] Crespo, A., Scherlis, D.A., Marti, M.A., Ordejon, P., Roitberg, A.E. and Estrin, D.A. (2003) A DFT based QM-MM approach designed for the treatment of large molecular systems: application to Chorismate mutase. J. Phys. Chem. B 49, 13728-13736.

[34] Perdew, J.P., Burke, K. and Ernzerhof, M. (1996) Generalized gradient approximation made simple. Phys. Rev. Lett. 18, 3865-3868.

[35] Marti, M.A., Scherlis, D.A., Doctorovich, F.A., Ordejon, P. and Estrin, D.A. (2003) Modulation of the NO trans effect in heme proteins: implications for the activation of soluble guanylate cyclase. J. Biol. Inorg. Chem. 8, 595-600.

[36] Capece, L., Estrin, D.A. and Marti, M.A. (2008) Dynamical characterization of the heme NO oxygen binding (HNOX) domain. Insight into soluble guanylate cyclase allosteric transition. Biochemistry 47, 9416-9427.

[37] Capece, L., Marti, M.A., Crespo, A., Doctorovich, F. and Estrin, D.A. (2006) Heme protein oxygen affinity regulation exerted by proximal effects. J. Am. Chem. Soc. $128,12455-12461$.

[38] Marti, M.A., Capece, L., Crespo, A., Doctorovich, F. and Estrin, D.A. (2005) Nitric oxide interaction with cytochrome $c^{\prime}$ and its relevance to guanylate cyclase. Why does the iron histidine bond break? J. Am. Chem. Soc. 127, 7721-7728.

[39] Case, D.A. et al. (2005) The Amber biomolecular simulation programs. J Comput. Chem. 26, 1668-1688.

[40] Berendsen, H.J.C., Postma, J.P.M., van Gunsteren, W.F., DiNola, A. and Haak, J.R. (1984) Molecular-dynamics with coupling to an external bath. J. Chem. Phys. 81, 3684-3690.

[41] Ryckaert, J.P., Ciccotti, G. and Berendsen, H.J.C. (1977) Numerical integration of the cartesian equations of motion of a system with constraints: molecular dynamics of $n$-alkanes. J. Comput. Phys. 23, 327-341.

[42] Sasaki, J., Imamura, T. and Yanase, T. (1978) Hemoglobin Hirose, a human hemoglobin variant with a substitution at the alpha1beta2 interface. Subunit dissociation and the equilibria and kinetics of ligand binding. J. Biol. Chem. 253, 87-94.

[43] Owen, M.C., Oekelford, P.A. and Wells, R.M.G. (1993) Hb Howick $[\beta 37(\mathrm{C} 3) \mathrm{TRP} \rightarrow \mathrm{GLY}]:$ a new high oxygen affinity variant of the $\alpha 1 \beta 2$ contact. Hemoglobin 17, 513-521.

[44] Hub, J.S., Kubitzki, M.B. and de Groot, B.L. (2010) Spontaneous quaternary and tertiary $\mathrm{T}-\mathrm{R}$ transitions of human hemoglobin in molecular dynamics simulation. PLoS Comput. Biol. 6, e1000774.

[45] Saito, M. and Okazaki, I. (2007) A 45-ns molecular dynamics simulation of hemoglobin in water by vectorizing and parallelizing COSMOS90 on the earth simulator: dynamics of tertiary and quaternary structures. J. Comput. Chem. 28, 1129-1136.

[46] Merlino, A., Fuchs, M.R., Pica, A., Balsamo, A., Dworkowski, F.S., Pompidor, G., Mazzarella, L. and Vergara, A. (2013) Selective X-ray-induced NO photodissociation in haemoglobin crystals: evidence from a Raman-assisted crystallographic study. Acta Crystallogr. D Biol. Crystallogr. 69, 137-140. 